\title{
Relativistic asymptotics of the deuteron and pion form factors
}

\author{
Alexander KRUTOV \\ Samara State University, Samara, Russia \\ E-mail: krutovessu.samara.ru \\ Vadim TROITSKY \\ D.V. Skobeltsyn Insitute of Nuclear Physics, Moscow, Russia \\ E-mail: troitsky@theory.sinp.msu.ru
}

\section{Natalia TSIROVA*}

Samara State University, Samara, Russia

E-mail: ntsirova@ssu.samara.ru

We present an investigation of the electromagnetic structure of the deuteron and the pion in a relativistic approach in the range of future JLab experiments. It is shown that this range could be considered as asymptotical for these systems. An asymptotical analysis of the deuteron and pion form factors is performed. We find that our relativistic calculations coincide with $\mathrm{pQCD}$ predictions if some restrictions are imposed.

LIGHT CONE 2008 Relativistic Nuclear and Particle Physics

July 7-11, 2008

Mulhouse, France

${ }^{*}$ Speaker. 


\section{Introduction}

We investigate the electromagnetic structure of composite systems in the framework of relativistic quantum mechanics (RQM) in the case of high momentum transfer. The motivation of our work is closely connected with the JLab $12 \mathrm{GeV}$ Upgrade program. One of the most important parts of this program is the investigation of the electromagnetic structure of composite systems at high momentum transfer [1]. At low energies the electromagnetic structure of such systems is described by well-known nonrelativistic methods. If energies increase, these methods fail and we should use relativistic ones. In the case of the highest energies, perturbative QCD (pQCD) should provide the most reliable description [2]. However, the question when pQCD starts to become valid is still open.

Here we investigate the electromagnetic structure of the deuteron as the simplest nucleonnucleon system and the pion as the simplest $q \bar{q}$ system. In future experiments of JLab the predicted behaviour of the form factors is expected to coincide with $\mathrm{pQCD}$ predictions for both the deuteron and the pion [1].

pQCD predictions are available for asymptotically high momentum transfers. So the region of future JLab experiment could be considered as asymptotical. That's why it is interesting to study the asymptotics of the deuteron and pion form factors. In this paper the asymptotical regime of the instant form of relativistic quantum mechanics $[3,4,5]$ is investigated for the deuteron and the pion.

\section{Instant form of relativistic quantum mechanics}

Let us describe briefly our model of relativistic quantum mechanics. In this section we also present the derivation of the electromagnetic pion form factor (see also [3]).

The charge form factor of a pion can be obtained from the electromagnetic current matrix element for a composite system in an arbitrary coordinate frame

$$
\left\langle p_{\pi}\left|j_{\mu}\right| p_{\pi}^{\prime}\right\rangle=\left(p_{\pi}+p_{\pi}^{\prime}\right)_{\mu} F_{\pi}\left(Q^{2}\right),
$$

$F_{\pi}\left(Q^{2}\right)$ - the electromagnetic form factor of the pion, describing the transition dynamics is an invariant function, the four-vector $\left(p_{\pi}-p_{\pi}^{\prime}\right)_{\mu}$ describes the geometric (transformation) properties of the matrix element, $p_{\pi}$ - the four-momentum of the pion.

In RQM the Hilbert space of composite particle states is the tensor product of single particle Hilbert spaces: $\mathscr{H}_{q \bar{q}} \equiv \mathscr{H}_{q} \otimes \mathscr{H}_{\bar{q}}$ and the state vector in RQM is a superposition of two-particle states. As a basis in $\mathscr{H}_{q \bar{q}}$ one can choose the following set of vectors:

$$
\begin{gathered}
\left|\vec{p}_{1}, m_{1} ; \vec{p}_{2}, m_{2}\right\rangle=\left|\vec{p}_{1}, m_{1}\right\rangle \otimes\left|\vec{p}_{1}, m_{2}\right\rangle, \\
\left\langle\vec{p}, m \mid \vec{p}^{\prime}, m^{\prime}\right\rangle=2 p_{0} \delta\left(\vec{p}-\vec{p}^{\prime}\right) \delta_{m m^{\prime}},
\end{gathered}
$$

Here $\vec{p}_{1}, \vec{p}_{2}$ - are particle momenta, $m_{1}, m_{2}$ - spin projections.

Since we consider the two-quark system as one composite system, the natural basis is one with separated center-of-mass motion:

$$
\left|\vec{P}, \sqrt{s}, J, l, S, m_{J}\right\rangle,
$$


with $P_{\mu}=\left(p_{1}+p_{2}\right)_{\mu}, P_{\mu}^{2}=s, \sqrt{s}-$ the invariant mass of two-particle system , $l-$ the angular momentum in the center-of-mass frame, $S$ - the total spin, $J$ - the total angular momentum, $m_{J}$ - the projection of the total angular momentum.

The basis (2.3) is connected with (2.2) through the Clebsch - Gordan decomposition of the Poincaré group [6].

Now the decomposition of the electromagnetic current matrix element for the composite system (2.1) in the basis (2.3) has the form

$$
\begin{aligned}
& \left(p_{\pi}+p_{\pi}^{\prime}\right)_{\mu} F_{\pi}\left(Q^{2}\right)=\sum \int \frac{d \vec{P}}{N_{C G}} \frac{d \vec{P}^{\prime}}{N_{C G}^{\prime}} d \sqrt{s} d \sqrt{s^{\prime}}\left\langle p_{\pi} \mid \vec{P}, \sqrt{s}, J, l, S, m_{J}\right\rangle \\
& \times\left\langle\vec{P}, \sqrt{s}, J, l, S, m_{J}\left|j_{\mu}\right| \vec{P}^{\prime}, \sqrt{s^{\prime}}, J^{\prime}, l^{\prime}, S^{\prime}, m_{J}{ }^{\prime}\right\rangle\left\langle\vec{P}^{\prime}, \sqrt{s^{\prime}}, J^{\prime},{ }^{\prime}, S^{\prime}, m_{J}{ }^{\prime} \mid p_{\pi}{ }^{\prime}\right\rangle .
\end{aligned}
$$

Here the sum is over the discrete variables of the basis (2.3). $\left\langle\vec{P}, \sqrt{s}, J, l, S, m_{J} \mid p_{\pi}\right\rangle$ is the composite system wave function,

$$
\left\langle\vec{P}^{\prime}, \sqrt{s^{\prime}}, J^{\prime}, l^{\prime}, S^{\prime}, m_{J}^{\prime} \mid p_{\pi}\right\rangle=N_{\pi} \delta\left(\vec{P}^{\prime}-\vec{p}_{c}\right) \delta_{J J^{\prime}} \delta_{m_{J} m_{J}^{\prime}} \delta_{l l^{\prime}} \delta_{S S^{\prime}} \varphi_{l S}^{J}(k) .
$$

$s=4\left(k^{2}+M^{2}\right), M$ is the quark mass, $N_{\pi}, N_{C G}$ are factors due to normalization. The concrete form of $N_{\pi}$ and $N_{C G}$ will not be used.

The basis (2.3) is the relativistic analogue of the basis of generalized spherical functions of nonrelativistic quantum mechanics (see, e.g. [7]). In this basis the pion wave function is the eigenfunction of the operators $\hat{J}^{2}, \hat{J}_{3}, \hat{l}^{2}$ as well as of the operator of the total spin squared $\hat{S}^{2}$, defined in an invariant way (see, e.g. [8]). All the operators have zero eigenvalues, because in the pion $J=l=S=0$. The quark spin properties are taken into account in the basis (2.3) by the corresponding Clebsh-Gordan decomposition.

In this approach we represent the pion electromagnetic form factor in the relativistic impulse approximation by the following integral form:

$$
F_{\pi}\left(Q^{2}\right)=\int d \sqrt{s} d \sqrt{s^{\prime}} \varphi(k) g_{0}\left(s, Q^{2}, s^{\prime}\right) \varphi\left(k^{\prime}\right) .
$$

Here $g_{0}\left(s, Q^{2}, s^{\prime}\right)$ is the so called free two-particle form factor to be derived by the methods of relativistic kinematics [6], $\varphi(k)$ is a phenomenological wave function normalized with account of the relativistic density of states [6]:

$$
\varphi(k)=\sqrt[4]{4\left(k^{2}+M^{2}\right)} k u(k), \quad \int d k k^{2} u^{2}(k)=1 .
$$

Here $u(k)$ is a nonrelativistic wave function.

While obtaining (2.6) we do not use a fixed coordinate frame (for example, Breit frame) or fixed ("good") current components, as one usually does in other RQM approaches [9]. In this respect our calculations are Lorentz-covariant. Our current matrix element satisfies conservation laws, so that the current operator of the composite system does not only contain the contribution of one-particle currents, but of two-particle currents too [6].

The deuteron form factors could be obtained in the same way. They have the following form:

$$
G_{C}\left(Q^{2}\right)=\sum_{l, l^{\prime}} \int d \sqrt{s} d \sqrt{s^{\prime}} \varphi_{l}(s) g_{0 C}^{l^{\prime}}\left(s, Q^{2}, s^{\prime}\right) \varphi_{l^{\prime}}\left(s^{\prime}\right),
$$




$$
\begin{aligned}
& G_{Q}\left(Q^{2}\right)=\frac{2 M_{d}^{2}}{Q^{2}} \sum_{l, l^{\prime}} \int d \sqrt{s} d \sqrt{s^{\prime}} \varphi_{l}(s) g_{0 Q}^{l l^{\prime}}\left(s, Q^{2}, s^{\prime}\right) \varphi_{l^{\prime}}\left(s^{\prime}\right), \\
& G_{M}\left(Q^{2}\right)=-M_{d} \sum_{l, l^{\prime}} \int d \sqrt{s} d \sqrt{s^{\prime}} \varphi_{l}(s) g_{0 M}^{l l^{\prime}}\left(s, Q^{2}, s^{\prime}\right) \varphi_{l^{\prime}}\left(s^{\prime}\right) .
\end{aligned}
$$

Here $\varphi_{l}(s), l, l^{\prime}=0,2$ are the deuteron wave functions in the sense of RQM, $g_{0 i}^{l l^{\prime}}, i=C, Q, M$ are the relativistic free two-particles charge, quadrupole, and magnetic form factors [10].

\section{Deuteron form factors asymptotics}

First, let us make some remarks about the deuteron wave functions used in (2.8). In modern calculations the deuteron wave functions usually are of the following analytic form in the momentum representation (see, e.g., Refs. [11, 12, 13]):

$$
u_{0}(k)=\sqrt{\frac{2}{\pi}} \sum_{j} \frac{C_{j}}{\left(k^{2}+m_{j}^{2}\right)}, \quad u_{2}(k)=\sqrt{\frac{2}{\pi}} \sum_{j} \frac{D_{j}}{\left(k^{2}+m_{j}^{2}\right)},
$$

or in the coordinate representation:

$$
u_{0}(r)=\sum_{j} C_{j} \exp \left(-m_{j} r\right), \quad u_{2}(r)=\sum_{j} D_{j} \exp \left(-m_{j} r\right)\left[1+\frac{3}{m_{j} r}+\frac{3}{\left(m_{j} r\right)^{2}}\right],
$$

here $m_{j}=\alpha+m_{0}(j-1), \alpha=\sqrt{M\left|\varepsilon_{d}\right|}, M$ is the average nucleon mass, $\varepsilon_{d}-$ the binding energy of the deuteron. The coefficients $C_{j}, D_{j}$, the maximal value of the index $j$ and $m_{0}$ are determined by the best fit of the corresponding solution of the Schrödinger equation.

The standard behavior of the functions at short distances:

$$
u_{0}(r) \sim r, \quad u_{2}(r) \sim r^{3},
$$

is provided by imposing the following conditions on the coefficients in (3.2):

$$
\sum_{j} C_{j}=0, \quad \sum_{j} D_{j}=\sum_{j} D_{j} m_{j}^{2}=\sum_{j} \frac{D_{j}}{m_{j}^{2}}=0 .
$$

Now let us turn to the asymptotics. In our paper [14] we obtain the asymptotic series for the deuteron form factors. The main asymptotic term for the deuteron form factor $F_{d}\left(Q^{2}\right)=\sqrt{G_{C}^{2}\left(Q^{2}\right)+\frac{8}{9} \eta^{2} G_{Q}^{2}\left(Q^{2}\right)+\frac{2}{3} \eta G_{M}^{2}\left(Q^{2}\right)}$ has the form:

$$
F_{d}\left(Q^{2}\right) \sim \frac{1}{\left(Q^{2}\right)^{4}}
$$

Let us compare the obtained asymptotic predictions with experimental data. Fitting the existing experimental points for the seven highest attained values of momentum transfer [15] in the region $3.040-5.955(\mathrm{GeV} / \mathrm{c})^{2}$ by a power-law function we obtain the following estimate for Eq. (3.5) with a normalized $\chi^{2}=1.34$ :

$$
F_{d}^{\exp }\left(Q^{2}\right) \sim \frac{1}{\left(Q^{2}\right)^{3.76 \pm 0.41}}
$$


So, comparing Eqs. (3.5) and (3.6) one can see that up to fitting accuracy, the experimental data are described by the relativistic formula (3.5).

Let us note that this result does not depend on the actual model of the $N N$-interaction and in fact is due to the general conditions (3.3) and (3.4) only. So, in the recent JLab experiments the range of momentum transfers, which can be characterized as an asymptotic one for our relativistic two-nucleon model of deuteron, is reached. Let us emphasize that this concerns our relativistic approach only. For example, in the relativistic approach of Ref. [16] the asymptotic decrease is faster than the experimental one and even faster than that of the quark-counting prediction.

Let us compare our results with those of the quark approach and of QCD in more detail. At $Q^{2} \rightarrow \infty$ there exists the well established prediction in the framework of these approaches $[17,18]$ :

$$
F_{d}\left(Q^{2}\right) \sim Q^{-10}
$$

As one can see, this prediction does not agree with the current experiment (3.6). So let us discuss the possibility to incorporate the QCD predictions in the nucleon-nucleon dynamics. We formulate the problem in the following way: what kind of behavior at small distances should the deuteron wave function have, or, in other words, how has the nucleon-nucleon potential at short distances to be modified, in order to obtain the asymptotic behavior of the electromagnetic deuteron form factors predicted by QCD? The answer can be obtained easily and the analysis shows that the quark-model asymptotic behavior could be derived in the nucleon dynamics formalism if in addition to the conditions (3.4) for the $s$-wave function (3.1) the following condition is imposed:

$$
\sum_{j} C_{j} m_{j}^{2}=0 \text {. }
$$

This condition means that in the vicinity of zero the wave function has the following form:

$$
u_{0}(r) \sim r+a r^{3}, u_{0}^{\prime \prime}(0)=0
$$

So, we have solved some kind of inverse problem: we found a condition for the deuteron wave function to give the asymptotic behavior of the deuteron electromagnetic form factors (in the framework of the two-nucleon model of deuteron) given by the quark approach.

\section{Pion form factor asymptotics}

The main asymptotic term for the electromagnetic pion form factor in the relativistic constituent quark model is of the following form:

$$
F_{\pi}\left(Q^{2}\right) \sim \frac{2^{5 / 2} M}{Q} \mathrm{e}^{-\frac{Q M}{4 b^{2}}}
$$

Here the quark mass $M$ and $b$ are parameters of the constituent quark model [3]. Let us note, that this formula is obtained for structureless quarks. In our relativistic approach it is possible to take into account quark structure by introducing quark form factors [3]. But quark form factors don't influence the decrease of the pion form factor, they just add logarithmic corrections. So in this paper we will work with structureless quarks. 
We also present the main asymptotic term in the small quark-mass limit:

$$
F_{\pi}\left(Q^{2}\right) \sim \frac{14 \sqrt{2} b^{2}}{Q^{2}}
$$

In $e \pi$ scattering in the case of low momentum transfer, the electron interacts not only with the constituent quarks but also with the sea-quarks cloud, so with an object of bigger mass. But if the energy is increased this cloud becomes smaller and smaller for the electron. So asymptotically high momentum tranfers lead us to asymptotically small quark masses. One can see that the main asymptotic term coincides with the pQCD predictions $\left(F_{\pi}\left(Q^{2}\right) \sim 1 / Q^{2}\right)$.

\section{Conclusion}

JLab predicts the pQCD-coinciding behavior of form factors of the deuteron and pion in future experiments. In our work we show that this behavior could be obtained in a relativistic two-nucleon model for the deuteron and a relativistic constituent-quark model for the pion with some restrictions.

\section{References}

[1] J. Arrington, R.J. Holt, P.E. Reimer et al., Hall A 12 GeV Upgrade. Pre-Conceptual Design Report, 2005.

[2] R. Gilman and F. Gross, J.Phys G. 28, R37 (2002).

[3] A.F. Krutov and V.E. Troitsky, Eur.Phys.J. C 20, 71 (2001).

[4] A. F. Krutov and V. E. Troitsky, Phys. Rev. C 65, 045501 (2002).

[5] A. F. Krutov and V. E. Troitsky, Phys. Rev. C 68, 018501 (2003).

[6] E.V. Balandina, A.F. Krutov, and V.E. Troitsky, Teor. Math. Phys. 103, 381 (1995).

[7] R.Newton, Scattering theory of waves and particles, 2nd ed., (Springer Verlag, New York, 1982).

[8] V.P. Kozhevnikov, V.E. Troitsky, S.V. Trubnikov, Yu.M. Shirokov, Theor.Math.Fiz. 10, 47, (1972).

[9] B.D. Keister and W. Polyzou, Adv.Nucl.Phys. 21, 225, (1991).

[10] A. F. Krutov and V. E. Troitsky, Phys. Rev. C 75, 014001 (2007).

[11] M. Lacombe, B. Loiseau, R. Vinh Mau, J. Coté, P. Pirés, R. de Tourreil, Phys. Lett. B 101, 139 (1981).

[12] R. Machleidt, Phys.Rev.C 63, 024001 (2001).

[13] A.F. Krutov and V.E. Troitsky, Phys. Rev. C 76, 017001 (2007).

[14] A. F. Krutov, V. E. Troitsky, and N. A. Tsirova, J. Phys. A: Math. Theor. 41, 255401 (2008).

[15] L.C. Alexa et al. (The Jefferson Lab Hall A Collaboration), Phys. Rev. Let. 82, 1374 (1999).

[16] R. G. Arnold, C. E. Carlson, and F. Gross, Phys.Rev. C 21, 1426 (1980).

[17] S. J. Brodsky and G. R. Farrar, Phys. Rev. Lett. 31, 1153 (1973).

[18] V. A. Matveev, R. M. Muradyan and A. N. Tavkhelidze, Lett. Nuovo Cim. 7, 719 (1973). 